\title{
Power corrections in charmless $B$ decays
}

\author{
T. N. Pham \\ Centre de Physique Théorique, \\ Centre National de la Recherche Scientifique, UMR 7644, \\ Ecole Polytechnique, 91128 Palaiseau Cedex, France
}

\begin{abstract}
Power corrections seem to play an important role in charmless $B$ decays as indicated by recent analysis using QCD Factorization. In this talk, I would like to report on a recent work on power corrections in charmless $B$ decays. By using the ratio of the branching fraction of $B^{+} \rightarrow \pi^{+} K^{* 0}$ to that of $B^{0} \rightarrow \pi^{-} \rho^{+}$, for which the theoretical uncertainties are greatly reduced, it is shown in a transparent manner that power corrections in charmless $B$ decays are probably large and that the $B^{0} \rightarrow K^{-} \rho^{+}$decay could be explained with the annihilation term included. For ratios of direct $\mathrm{CP}$ asymmetries, QCD Factorisation with the annihilation terms included would predict the direct $\mathrm{CP}$ asymmetry of $B \rightarrow \pi^{+} \pi^{-}$to be about 3 times larger than that of $B \rightarrow \pi^{ \pm} K^{\mp}$, with opposite sign. In particular, the large measured value for $B \rightarrow \pi^{ \pm} K^{\mp} \mathrm{CP}$ asymmetry implies naturally a corresponding large $B \rightarrow \pi^{+} \pi^{-}$CP asymmetry as observed by Belle. Experimentally any significant deviation from this prediction would suggest either new physics or possibly the importance of long-distance rescattering effects.
\end{abstract}

PC061.1104

November 2004

$\dagger$ Talk given at the QCD Euroconference 2004, Montpellier 5-9 July 2004 


\section{Introduction}

In QCD Factorization (QCDF)[1], the $O\left(1 / m_{b}\right)$ power corrections in penguin matrix elements and other chirally enhanced corrections could make important contributions to the penguin-dominated charmless $B$ decays as in $B \rightarrow \pi K$ decays. Other power corrections terms such as annihilation contributions may also be present in PP and PV decays as first noticed in the perturbative QCD method for charmless $B$ decays[2] and indicated by recent analysis of charmless two-body non-leptonic $B$ decays $[3,4,5]$. In a recent work $[6]$, we have shown that in $\mathrm{QCDF}$, it is possible to consider certain ratios of the $B \rightarrow P V$ amplitudes which depend only on the Wilson coefficients and the known hadronic parameters. The discrepancy between prediction and experiment for the ratio would be a clear evidence for annihilation or other non factorisable contributions. We find that annihilation topology likely plays an indispensable role at least for penguin-dominated PV channels. Including the annihilation terms in QCDF, we find that the direct CP asymmetry of $B \rightarrow \pi^{+} \pi^{-}$to be about 3 times larger than that of $B \rightarrow K^{\mp} \pi^{ \pm}$, with opposite sign, in agreement with experiment.

\section{QCD factorization for charmless $B$ decays}

The effective Lagrangian for non-leptonic $B$ decays can be obtained from operator product expansion and renormalization group equation, in which short-distance effects involving large virtual momenta of the loop corrections from the scale $M_{W}$ down to $\mu=\mathcal{O}\left(m_{b}\right)$ are integrated into the Wilson coefficients. The amplitude for the decay $B \rightarrow M_{1} M_{2}$ can be expressed as

$$
\mathcal{A}\left(B \rightarrow M_{1} M_{2}\right)=\frac{G_{F}}{\sqrt{2}} \sum_{i=1}^{6} \sum_{q=u, c} \lambda_{q} C_{i}(\mu)\left\langle M_{1} M_{2}\left|O_{i}(\mu)\right| B\right\rangle
$$

$\lambda_{q}$ is a CKM factor, $C_{i}(\mu)$ are the Wilson coefficients perturbatively calculable from first principles and $O_{i}$ are the tree and penguin operators given by(neglecting other operators):

$$
\begin{aligned}
& O_{1}=(\bar{s} u)_{L}(\bar{u} b)_{L} \quad, O_{4}=\sum_{q}(\bar{s} q)_{L}(\bar{q} b)_{L} \\
& O_{6}=-2 \sum_{q}\left(\bar{s}_{L} q_{R}\right)\left(\bar{q}_{R} b_{L}\right)
\end{aligned}
$$

The hadronic matrix elements : $\left\langle M_{1} M_{2}\left|O_{i}(\mu)\right| B\right\rangle$ contains the physics effects from the scale $\mu=\mathcal{O}\left(m_{b}\right)$ down to $\Lambda_{\mathrm{QCD}}$. In the heavy quark limit, QCD Factorisation [1] allows the decay mplitude $\left\langle M_{1} M_{2}\left|O_{i}(\mu)\right| B\right\rangle$ to be factorized into hard radiative corrections and non perturbative matrix elements which can be parametrized by the semi-leptonic decays form factors and meson light-cone distribution amplitudes (LCDAs).

Power corrections in $1 / m_{b}$ come from penguin matrix elements, chirally enhanced corrections and annihilation contributions. For example, in the $B \rightarrow \pi K$ amplitude, the matrix element of $O_{6}$ is of the order $O\left(1 / m_{b}\right)$ compared to the $(V-A) \times(V-A) O_{1}$ and $O_{4}$ matrix 
elements, since $<K\left|\bar{s}_{L} d_{R}\right| 0>$ is proportional to $m_{K}^{2} / m_{s} \approx 2.5 \mathrm{GeV}$ while $<K\left|\bar{s}_{L} d_{L}\right| 0>$ is proportional to $K$ momentum which is $O\left(m_{b}\right)$, thus numerically, the matrix element of $O_{6}$ which has a factor

$$
r_{\chi}^{K}=\frac{2 m_{K}^{2}}{m_{b}\left(m_{s}+m_{d}\right)} \approx O(1)
$$

is comparable to that of $O_{4}$. For penguin-dominated, decays, the $O_{4}$ and $O_{6}$ matrix element are of the same sign in PP channnel, while in PV channel they are of opposite sign. Thus in QCDF one expects a small $B \rightarrow K \rho$ branching ratio relative to $B \rightarrow \pi K$. Because of cancellation between the $O_{4}$ and $O_{6}$ contributions, the $B \rightarrow K \rho$ decay is more sensitive to other power corrections and non factorisable contributions. Including the chirally-enhanced corrections in terms of two quantities $X_{\mathrm{A}, \mathrm{H}}$ and a strong phase, the $B \rightarrow M_{1} M_{2}$ decay amplitudes in QCDF can be thus be written as[7, 8]:

$$
\mathcal{A}\left(B \rightarrow M_{1} M_{2}\right)=\frac{G_{F}}{\sqrt{2}} \sum_{p=u, c} V_{p b} V_{p s}^{*}\left(-\sum_{i=1}^{6} a_{i}^{p}\left\langle M_{1} M_{2}\left|O_{i}\right| B\right\rangle_{f}+\sum_{j} f_{B} f_{M_{1}} f_{M_{2}} b_{j}\right),
$$

\section{Power corrections in $B \rightarrow \mathrm{PV}$ decays}

Consider the ratio of $A\left(B^{+} \rightarrow \pi^{+} K^{* 0}\right)$ to $A\left(B^{0} \rightarrow \rho^{+} \pi^{-}\right)$. amplitudes. If the power corrections were negligible, this ratio would be theoretically very clean where the form factors cancel out, furthermore it is almost independent ot the CKM angle $\gamma$ and the strange-quark mass:

$$
\left|\frac{\mathcal{A}\left(B^{+} \rightarrow \pi^{+} K^{* 0}\right)}{\mathcal{A}\left(B^{0} \rightarrow \rho^{+} \pi^{-}\right)}\right| \simeq\left|\frac{V_{c b} V_{c s}}{V_{u b} V_{u d}}\right| \frac{f_{K^{*}}}{f_{\rho}}\left|\frac{a_{4}^{c}\left(\pi K^{*}\right)+r_{\chi}^{K^{*}} a_{6}^{c}\left(\pi K^{*}\right)}{a_{1}^{u}}\right|
$$

$$
\left|\left(a_{4}^{c}\left(\pi K^{*}\right)+r_{\chi}^{K^{*}} a_{6}^{c}\left(\pi K^{*}\right)\right) / a_{1}^{u}\right| \text { should be about or less than } 0.04 \text { in QCDF. }\left(f_{K^{*}} / f_{\rho} \approx 1\right) .
$$

The ratio $\left|V_{u b} / V_{c b}\right|$ is not very well determined experimentally, but a stringent lower limit can be obtained from the unitarity of the CKM matrix . Since $[9,10]$ :

$$
\left|\frac{V_{u b}}{V_{c b}}\right|=\lambda \sin \beta \sqrt{1+\frac{\cos ^{2} \alpha}{\sin ^{2} \alpha}} \geq \lambda \sin \beta .
$$

and from the current Babar and Belle measured values $\sin 2 \beta=0.736 \pm 0.049$ [11], we have

$$
\left|\frac{V_{u b}}{V_{c b}}\right| \geq \lambda \sin \beta=0.090 \pm 0.007>0.078
$$

Eq.(5) implies the following inequality :

$$
0.53>\left|\frac{\mathcal{A}\left(B^{+} \rightarrow \pi^{+} K^{* 0}\right)}{\mathcal{A}\left(B^{0} \rightarrow \rho^{+} \pi^{-}\right)}\right|=0.77 \pm 0.09
$$

where the number on the rhs is from the measured branching ratios [12, 13], . The lhs would be reduced further to $0.46 \pm 0.04$, if one neglects a small $\cos ^{2} \alpha$ term in Eq.(6).

Since the chirally enhanced corrections for penguin-dominated decays are not expected to be large, this large discrepancy is strong indication that annihilation topology and/or 


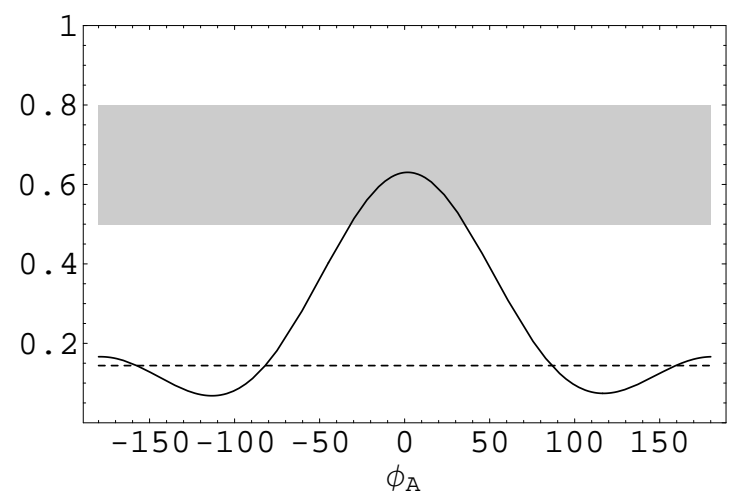

Figure 1: The ratio $\mathcal{B}\left(B^{+} \rightarrow \pi^{+} K^{* 0}\right) / \mathcal{B}\left(B^{0} \rightarrow \rho^{+} \pi^{-}\right)$versus the weak annihilation phase $\phi_{A}$. The default parameters are used but letting the annihilation parameter $\rho_{A}=1$. The dashed lines show the ratios without weak annihilation contributions. The gray areas denote the experimental measurements with $1 \sigma$ error.

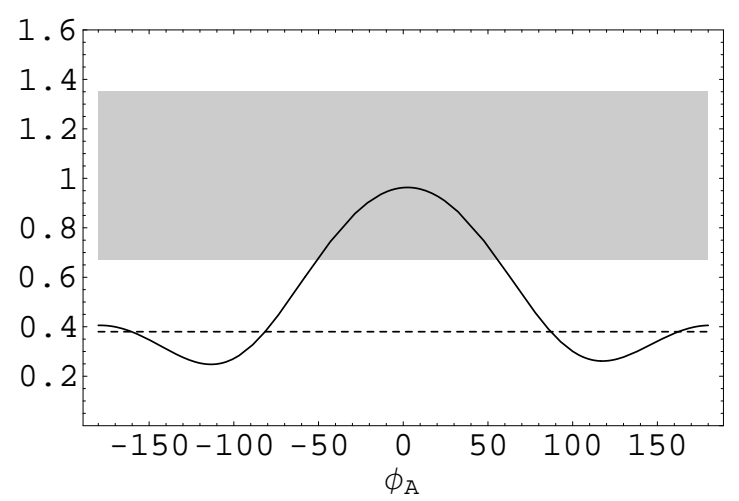

Figure 2: The ratio $\mathcal{B}\left(B^{0} \rightarrow K^{+} \rho^{-}\right) / \mathcal{B}\left(B^{0} \rightarrow \rho^{-} \pi^{+}\right)$versus the weak annihilation phase $\phi_{A}$. The default parameters are as in Fig.1

other sources of power corrections might play an important role at least in $B \rightarrow \mathrm{PV}$ decays. There is similar disagreement between theory and experiment in another ratio, the branching fraction of $B^{0} \rightarrow K^{+} \rho^{-}$to that of $B^{0} \rightarrow \rho^{-} \pi^{+}$, though with large theoretical uncertainties. For $\gamma=70^{\circ}, V_{u b} / V_{c b}=0.09, a_{4}^{c}(\rho K)-r_{\chi}^{K} a_{6}^{c}(\rho K)=0.037+0.003 i, m_{s}=90 \mathrm{MeV}$, we find

$$
\frac{\mathcal{B}\left(B^{0} \rightarrow K^{+} \rho^{-}\right)}{\mathcal{B}\left(B^{0} \rightarrow \rho^{-} \pi^{+}\right)}=0.38
$$

far below the measured value of $1.01 \pm 0.34$, though, this ratio could be increased to 0.69 , if $m_{s}$ is lowered to $70 \mathrm{MeV}$.

Taken together, these results indicate that the penguin-dominated $B \rightarrow \mathrm{PV}$ decay amplitudes are consistently underestimated without annihilation contributions. Including the annihilation terms, from Eq. (4), we have 


$$
\begin{aligned}
& A\left(B^{+} \rightarrow \pi^{+} K^{* 0}\right)=f_{K^{*}} F^{B \pi} m_{B}^{2} a_{4}+b_{3}(V, P) \\
& A\left(B^{0} \rightarrow K^{+} \rho^{-}\right)=f_{K} A_{0}^{B \rho} m_{B}^{2}\left(a_{4}-r_{\chi}^{K} a_{6}\right)+b_{3}(P, V) \\
& b_{3}\left(M_{1}, M_{2}\right)=\frac{C_{F}}{N_{c}^{2}}\left\{C_{3} A_{1}^{i}\left(M_{1}, M_{2}\right)+C_{5} A_{3}^{i}\left(M_{1}, M_{2}\right)+\left(C_{5}+N_{c} C_{6}\right) A_{3}^{f}\left(M_{1}, M_{2}\right)\right\}
\end{aligned}
$$

With the penguin terms $a_{4} \simeq-0.03$ and $a_{4}-r_{\chi}^{K} a_{6} \simeq 0.037$ having opposite sign, the key observation is that $b_{3}(V, P)$ and $b_{3}(P, V)$, which get most of the contribution from $\left(C_{5}+N_{c} C_{6}\right) A_{3}^{f}$ term, are also roughly of the opposite sign since $A_{3}^{f}(P, V)=-A_{3}^{f}(V, P)$. Thus QCDF can easily enhance both ratios without fine tuning ( no large strong phase ) as can be seen in Fig. 2 .

\section{Direct CP violations}

We now turn to the $\mathrm{CP}$ asymmetries in QCDF with annihilation terms included. Because of the CKM factor and $S U(3)$ symmetry for the tree and penguin matrix elements in $B^{0} \rightarrow$ $\pi^{+} \pi^{-}$and $B^{0} \rightarrow K^{+} \pi^{-}$decays, one can derive a relation between direct CP asymmetries in these two channels. With the CP asymmetry given as:

$$
\begin{aligned}
& A_{\pi \pi}=\frac{4\left|V_{u b} V_{u d} V_{c b} V_{c d} T_{\pi \pi} P_{\pi \pi}\right| \sin \gamma \sin \delta}{2 \mathcal{B}\left(B \rightarrow \pi^{+} \pi^{-}\right)}, \\
& A_{\pi K}=-\frac{4\left|V_{u b} V_{u s} V_{c b} V_{c s} T_{\pi K} P_{\pi K}\right| \sin \gamma \sin \tilde{\delta}}{2 \mathcal{B}\left(B \rightarrow \pi^{+} K^{-}\right)} .
\end{aligned}
$$

( $\delta=\delta_{P}-\delta_{T}=$ strong phases difference between the penguin and tree amplitudes), we find

$$
\begin{aligned}
\frac{A_{\pi \pi}}{A_{\pi K}} & =-\frac{f_{\pi}^{2}}{f_{K}^{2}} \frac{\mathcal{B}\left(B \rightarrow \pi^{+} K^{-}\right)}{\mathcal{B}\left(B \rightarrow \pi^{+} \pi^{-}\right)}\left|\frac{T_{\pi \pi} P_{\pi \pi}}{T_{\pi K} P_{\pi K}}\right| \frac{\sin \delta}{\sin \tilde{\delta}} \\
& \simeq(-2.7 \pm 0.3) \frac{\sin \delta}{\sin \tilde{\delta}}
\end{aligned}
$$

a consequence of the fact that $T_{\pi \pi} P_{\pi \pi} / T_{\pi K} P_{\pi K}$ is close to 1 , a reasonable approximation in QCDF, at about 10 percent level uncertainty. A previous derivation of this relation is given in [16]. Belle has claimed large direct $\mathrm{CP}$ asymmetry observed in $B^{0} \rightarrow \pi^{+} \pi^{-}$decay while BaBar has not confirmed it yet, but both of them are close to a measurement on $A_{C P}\left(\pi^{-} K^{+}\right)[14,15]$

$$
\begin{aligned}
& A_{\pi \pi}= \begin{cases}0.58 \pm 0.15 \pm 0.07 & (\text { Belle }) \\
0.19 \pm 0.19 \pm 0.05 & (\text { BaBar })\end{cases} \\
& A_{\pi K}= \begin{cases}(-8.8 \pm 3.5 \pm 1.8) \% & \text { (Belle) } \\
(-13.3 \pm 3.0 \pm 0.9) \% & (\text { BaBar })\end{cases}
\end{aligned}
$$

We thus expect very naturally a larger direct CP violation for $\pi^{+} \pi^{-}$decay compared with $\pi^{-} K^{+}$decay, since the $\pi^{+} \pi^{-}$decay rate is smaller than the $\pi^{-} K^{+}$decay rate by factor $3-4$, 
Experimentally,

$$
\frac{A_{\pi \pi}}{A_{\pi K}}=\frac{0.42 \pm 0.13}{-0.11 \pm 0.03}=-4.0 \pm 1.8
$$

still consistent with the theoretical estimation of $-2.7 \pm 0.3$.

Similar relation between $\mathrm{CP}$ asymmetries for the $B \rightarrow P V$ decays for which the CPviolating interference terms are essentially of the same magnitude, but with opposite sign:

$$
\begin{aligned}
& \frac{A_{\mathrm{CP}}\left(B^{0} \rightarrow \rho^{+} \pi^{-}\right)}{A_{\mathrm{CP}}\left(B^{0} \rightarrow K^{*+} \pi^{-}\right)} \simeq-\frac{\mathcal{B}\left(B^{0} \rightarrow K^{*+} \pi^{-}\right)}{\mathcal{B}\left(B^{0} \rightarrow \rho^{+} \pi^{-}\right)} \frac{f_{\rho}^{2}}{f_{K^{*}}^{2}} \frac{\sin \delta_{\pi \rho}}{\sin \delta_{\pi K^{*}}} \\
& \frac{A_{\mathrm{CP}}\left(B^{0} \rightarrow \rho^{-} \pi^{+}\right)}{A_{\mathrm{CP}}\left(B^{0} \rightarrow \rho^{-} K^{+}\right)} \simeq-\frac{\mathcal{B}\left(B^{0} \rightarrow \rho^{-} K^{+}\right)}{\mathcal{B}\left(B^{0} \rightarrow \rho^{-} \pi^{+}\right)} \frac{f_{\pi}^{2}}{f_{K}^{2}} \frac{\sin \delta_{\rho \pi}}{\sin \delta_{\rho K}}
\end{aligned}
$$

\section{Conclusion}

Power corrections in charmless B decays are probably large, at least for the penguindominated PV channel. The key observation is that QCDF predicts the annihilation terms for $B^{+} \rightarrow \pi^{+} K^{* 0}$ and $B^{0} \rightarrow K^{+} \rho^{-}$to be almost equal in magnitude but opposite in sign and thus enhance the decay rates for these two modes to accommodate the experimental data. The relation for the direct CP asymmetry would naturally implies a large CP asymmetry for $B \rightarrow \pi^{+} \pi^{-}$, about 3 times larger than that of $B \rightarrow \pi^{ \pm} K^{\mp}$ with opposite sign.

I would like to thank S. Narison and the organisers of QCD04 for the warm hospitality extended to me at Montpellier.

\section{References}

[1] M. Beneke, G. Buchalla, M. Neubert and C.T. Sachrajda, Phys. Rev. Lett. 83 (1999) 1914 ; Nucl. Phys. B 591 (2000) 313.

[2] Y.Y. Keum, H.N. Li and A.I. Sanda, Phys. Rev. D 63, 054008 (2001) ; Phys. Lett. B $504(2001) 6$.

[3] D.S. Du, J.F. Sun, D.S. Yang and G.H. Zhu, Phys. Rev. D 67 (2003) 014023.

[4] R. Aleksan, P.F. Giraud, V. Morenas, O. Pene and A.S. Safir, Phys. Rev. D 67 (2003) 094019.

[5] N.de Groot, W.N. Cottingham and I.B. Whittingham, Phys. Rev. D 68, (2003) 113005.

[6] T. N. Pham and Guohuai Zhu, Phys.Rev. D 69 (2004) 114016.

[7] M. Beneke, G. Buchalla, M. Neubert and C.T. Sachrajda, Nucl. Phys. B 606 (2001) 245 .

[8] M. Beneke and M. Neubert, Nucl. Phys. B 675 (2003) 333 . 
[9] T.N. Pham, invited talk at 2nd Workshop on the CKM Unitarity Triangle, Durham, April 2003, hep-ph/0306271.

[10] A.J. Buras, F.Parodi and A. Stocchi, JHEP 0301 (2003) 029 .

[11] J. Alexander, P. Chang and J. Smith, Heavy Flavor Averaging Group, September 2003.

[12] H. Evans, A. Höcker, O. Long, M. Shapiro and Y. Sakai, Heavy Flavor Averaging Group, Summer 2003.

[13] A. Höcker, M. Laget, S. Laplace and J.V. Wimmersperg-Toeller, Using Flavor Symmetry to Constraint $\alpha$ from $B \rightarrow \rho \pi$, preprint LAL 03-17.

[14] Belle Collaboration, K. Abe et al., hep-ex/0401029; T. Tomura et al. , hep-ex/0305036.

[15] BaBar Collaboration, B.Aubert et al hep-ex/0407057.

[16] R. Fleischer, Phys. Lett. B 459 (1999) 306. 